\title{
Information Processing for Remote Sensing
}

Ed. Chen CH, World Scientific, New Jersey (1999)

\section{DISCRIMINATION OF BURIED PLASTIC AND METAL OBJECTS IN SUBSURFACE SOIL}

\author{
D. C. CHIN, DR. R. SRINIVASAN, AND ROBERT E. BALL \\ Johns Hopkins University Applied Physics Laboratory, Johns Hopkins RD., Laurel, MD 20723 \\ E-mail: laniel.chin@jhuapl.edu
}

\begin{abstract}
The Electrical Conductivity Object Locator (ECOL) has been developed with the goal of detecting buried objects. Its specific capability to detect and characterize small-size plastic and metal objects buried at shallow depths is demonstrated. The technique can also detect larger objects at greater depths. The ECOL technique maps the soil subsurface conductivity and identifies variations in the conductivity between buried objects and their surroundings. The subsurface conductivity is mapped in two major steps: 1) Low-frequency (1 to $100 \mathrm{~Hz})$ and low-amplitude $(<200 \mu \mathrm{A})$ currents injected into the soil induce potential and magnetic fields in and around the subsurface soil. The potential and magnetic fields are measured using appropriate sensors placed above the soil surface. 2) Using the measured values as boundary conditions, a fast optimization algorithm, and an accurate matrix inversion routine, the subsurface conductivity is estimated. The ECOL technology has been verified extensively through computer simulation and field tests. Computer simulations were conducted using small and large plastic and metal objects buried at various depths between 10 and 50 meters; field-tests were conducted using small objects buried at shallow depths. They indicate that the ECOL technique is able to identify buried plastic discs $(20 \mathrm{~cm}$ diameter; $5 \mathrm{~cm}$ thick) or metal discs (15 cm diameter, $7 \mathrm{~cm}$ thick) at shallow depths of about $15 \mathrm{~cm}$. Note that during the field tests, the soil was cluttered with roots and pebbles. The moisture content of the soil did not affect the ability to locate buried objects. During the field tests, conventional sensors, such as reference "half-cells" were used in measuring electrical potentials. The subsurface conductivity was reconstructed using a set of finite elements and a simultaneous stochastic perturbation approximation algorithm, which were specifically developed for this technique. The codes were validated through extensive computer simulation of the experimental conditions.
\end{abstract}

\section{Introduction}

The Electrical Conductivity Object Locator (ECOL) uses electric conductivity maps to distinguish buried foreign objects from the regular soil in the subsurface. Assuming that foreign objects and the regular soil have different electrical conductivities, when an electrical current is induced into the subsurface, the difference in conductance causes an electrical field distortion. Theoretically, one can measure the outside field distortion to solve the conductivity profile. Because the problem is highly nonlinear and field measurements are noisy, mapping the conductivity profile is an interesting and challenging task. In addition, the high contrasts in conductivity values among metallic and nonmetallic objects and soil and the high correlation within the model parameters add to the level of difficulty. The high contrast causes computational instability in the inversion; the high correlation is due to locating the small objects. The ECOL technology utilizes several mathematical techniques that the Johns Hopkins University Applied Physics Laboratory (JHU/APL) has developed in the past to overcome the difficulties and locate the mine-like small objects.

ECOL applies a low-amplitude $(\sim 200-\mu \mathrm{A})$ electric alternating current, single or multiple frequency. The impressed AC current generates AC potentials and magnetic fields throughout the site, which are measured at the surface and the boundary of the site. Also, ECOL establishes a finite element model to compute the surface and boundary values from the amount of current, physical structure, and an initial estimate of the conductivity profile for the subsurface. ECOL updates the estimate of the conductivity profile of the subsurface and the properties of the buried object by minimizing the sum of 
the square of the differences between the measured and the computed values, namely the differential potentials at the boundary. The minimization is based on a gradient approximation technique, namely, simultaneous perturbation stochastic approximation (SPSA) [1,2]. A finite element method (FEM) is used to compute the potentials or magnetic field from a known or an estimated conductivity profile.

\section{Background}

Subsurface conductivity measurements, sometimes termed conductivity-tomography or electrical-impedance-tomography, have been reported in the published literature since the late $1970 \mathrm{~s}[3,4,5$, and 6]. These measurements have been widely used in mapping the internal conductivity of biological bodies, as well as geological sites. These techniques involve placing electrodes around the site that contains objects under investigation and sending a known amount of electrical current through the site. The current generates electrical potentials or fields that are measured using another set of electrodes placed on the surface. Next, the internal conductivity of the object is reconstructed using the measured potential values, injected current, and the location of the electrodes. Several types of mathematical reconstruction algorithms have been used and reported since 1970s [3, 4, 5, and 6]. Most of these techniques have attained a certain level of qualified success: the subsurface conductivity can be mapped (1) when the differences in the conductivity values between the site elements and objects are small and (2) when the internal structure and the substance is well defined [7]. However, none of these techniques has been successful in locating small plastic or metal mines buried at shallow depths.

The ECOL technique maps and creates images of the conductivity profiles of the subsurface in the region of interest by injecting electrical currents into the soil. It is a noninvasive technique that works in five major steps (see Fig. 1). Steps 1 and 2 provide the experimental data needed for the reconstruction procedure, described in Steps 3 through 5, that locates and characterizes the buried object.

Step 1: Electrodes are placed around the site and connected to a power source. An electric current is injected into the site (Block 1 in Fig. 1). The electric current generates electrical potentials in the entire site, including the surface.

Step 2: The electrical potentials generated in Step 1 are measured using "reference electrodes" placed at the surface of the soil (Block 2 in Fig. 1).

Step 3: The subsurface of the site is divided into many elements. Each element is given an initial arbitrary conductivity value (Block 3 in Fig. 1).

Step 4: This step consists of two parts. Step 4a constructs a perturbation array from a random set of numbers generated from a Bernoulli distribution with outcome \pm 1 and scaled by a step gain constant. (The choice of the step gain constant is according to the requirements of SPSA.) Then, two perturbed conductivity profiles are created by adding and subtracting the perturbation array from a set of previously estimated or assumed parameters (Block 4a in Fig. 1). Step 4b computes voltage potentials for every element within the soil subsurface from the perturbed conductivity profiles using an FEM-bound algorithm (Block $4 \mathrm{~b}$ in Fig. 1).

Step 5: The SPSA algorithm compares the computed boundary values, the differential potentials, from Step $4 \mathrm{~b}$ with the measured values from Step 2 to approximate the gradients that update the parameters. Finally, if the termination criteria are not reached, it returns to Step 4 for the next iteration (Block 5 in Fig. 1).. 


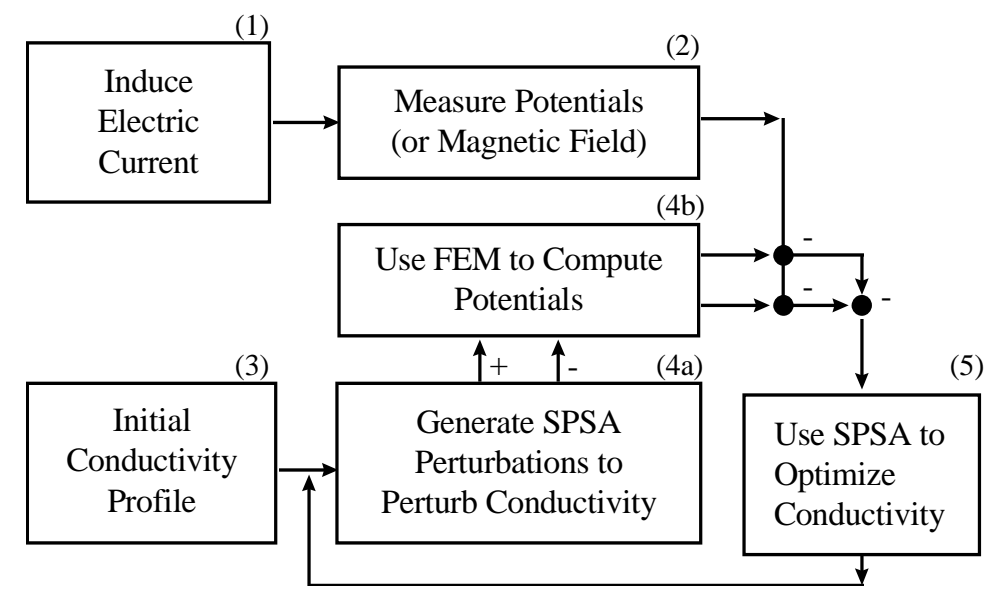

Figure 1: The Configuration of ECOL

The "+" and "-" signs in Fig. 1 represent mathematical operations as they are described in the steps. In a simulation, the first two steps would be replaced by the boundary potentials computed from an assumed conductivity profile; the last three steps remain unchanged

\section{Reconstruction of the Internal Conductivity of the Mine Location}

This section presents a brief description of the procedure that generates the soil subsurface conductivity map. A formal description of two important aspects of the reconstruction process was described in Step 4a (SPSA) and Step 4b (FEM) in the Methodology section. A complete discussion of FEM can be found in [8]. The reconstruction process solves the generalized Laplace equations mixed with Dirichlet and Neumann boundary conditions. This equation is called the Galerkin's error minimization method. A two-dimensional FEM model of the experimental site for computing the potentials is shown in Fig. 2. It represents a cross section of the subsurface $100 \mathrm{~cm}$ deep by $500 \mathrm{~cm}$ wide. The subsurface is divided into two sets of small divisions (rectangular blocks in different sizes). Each division is called an element in FEM. The center region (50 by $90 \mathrm{~cm}$ ) is the region of interest and consists of 45 equal-size rectangular elements. The gray shaded region located outside the center region is the region of influence that consists of 99 unequal-size rectangular elements.

Surface

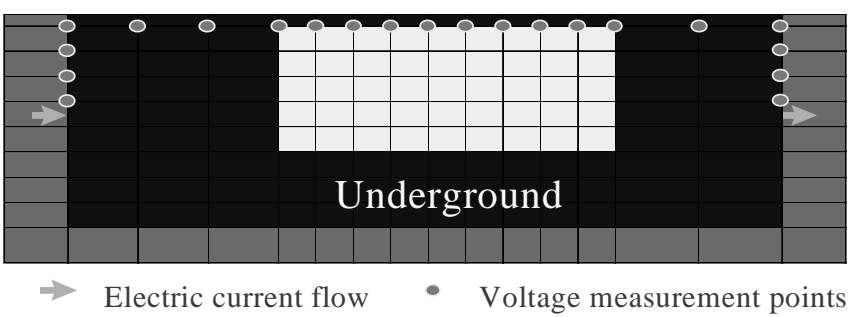

Figure 2: Finite Element Model for Field Demonstrations 
Steps 3 through 5 (see Methodology) follow the SPSA algorithm. Note that ECOL follows two distinct parametric approaches, one (defined by [2]) for estimating the conductivity of the elements in the $50-$ by $90-\mathrm{cm}$ middle section, and the other for the elements in the influence region outside the middle section. The conductivity values of the elements in the latter section are not optimized; they are kept at the same values throughout the iterations. The loss function of the SPSA algorithms is the sum of the square differences of the measured potential differences versus the FEM computed differences. The gain sequence used in SPSA is modified to avoid multiple solutions; the gain sequence $\{c\}$ defined in [1] was kept as a stepwise reduction (the value changed every 30 iterations). Discussion of the global optimization algorithm can be found in [9].

\section{Field Demonstrations}

The applicability of ECOL to locate mine-sized objects was demonstrated through two separate sets of field tests. The setting is shown in Fig. 3. In Test 1, a 20-inch-diameter by 5 -cm-thick plastic object was buried and located at two separate times. First, it was located 30 minutes after burial, and the second time it was located about 5 months after burial. In Test 2, a 10-cm-diameter by 7-cm-thick metal object was buried and located 30 minutes after burial. In both cases, the soil was not specially modified for testing or demonstrating purposes; other items, including roots, pebbles, and plants, present in the site were left mostly undisturbed. The test objects were buried only 10 to $15 \mathrm{~cm}$ below the soil surface. The total area of the site tested was approximately $300 \mathrm{~cm}$ by $300 \mathrm{~cm}$. The results of the tests are described below.

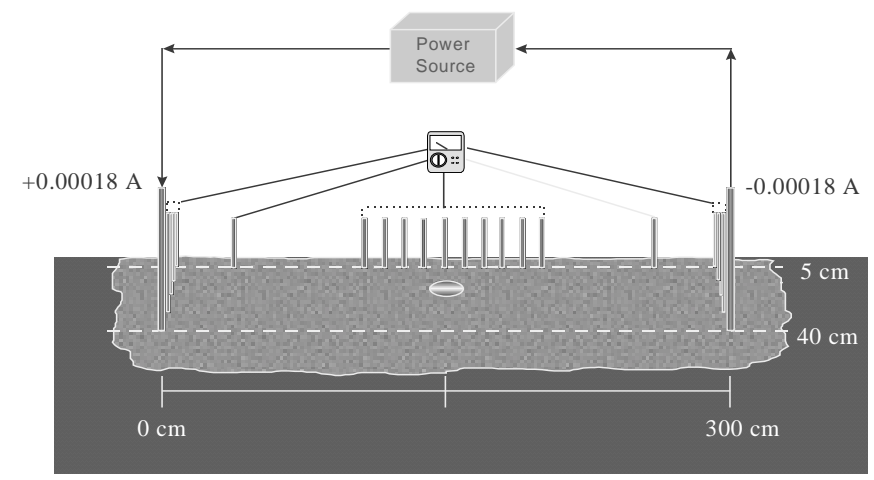

Figure 3: Diagram of the Demonstration Site

Figs. 4 and 5 show the reconstructed subsurface conductivity for the field experiments; only the middle section around the location of the object is shown. Fig. 4 shows the results from the plastic object that was buried between the fourth and fifth elements from the left on the second row of the figure and occupied only a fraction of both elements. Note that the low-conductivity value representing a plastic-like object is clearly identifiable. The estimated location is $5 \mathrm{~cm}$ left of the buried site and is omitted from the figure. Fig. 5 shows the results from the metal object, which was buried at the same location as the plastic object. Note that the conductivity of the soil, which is much higher than the plastic object in Fig. 3 (or much lower than the metal object in Fig. 4), is nonuniform. We attribute this to the presence of pebbles and roots in the soil. The numbers shown in both figures emerged after only 100 iterations and took less than 5 minutes in a Pentium-equipped computer. 


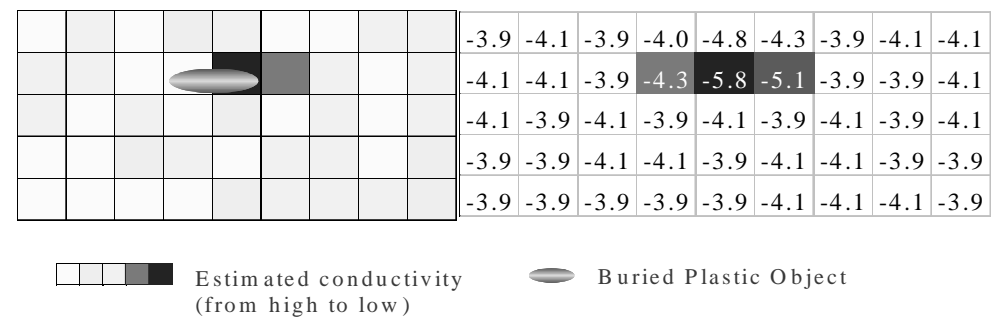

Figure 4: Reconstruction of the Conductivities for Test 1. (The table entries are in $\log$ scale)
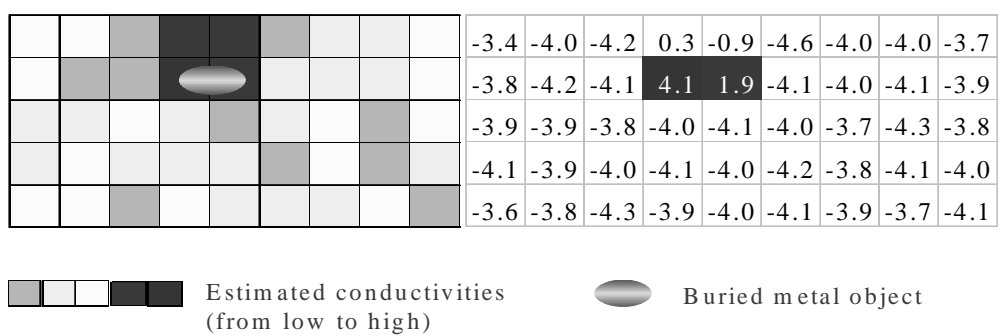

\section{Figure 5: Reconstruction of the Conductivities for Test 2 (the table entries are in log scale.)}

Note that these experiments were conducted under a virtual blindfold condition, in which the algorithm made no a priori assumption about either the character of the objects or the conductivity of the soil.

\section{Conclusions}

The purpose of the Electrical Conductivity Object Locator is to generate an internal map of the location, size, and conductivity of all objects in a suspected site having plastic and/or metal mines. In this work, we have demonstrated that the ECOL technique is able to locate small-sized plastic and metal objects buried in shallow depths in cluttered soil.

The ECOL technique assumes spatial nonuniformity for conductivity of the soil subsurface. It divides the subsurface space into several elements and assumes that the objects of interest are present within some of those elements. The technique injects a small-amplitude, low frequency electrical current into the soil and measures the resulting electrical potentials at the soil surface. The conductivity of each element of the subsurface is reconstructed using the injected current as the input parameter and the measured potentials as the boundary condition. A sequence of algorithms, all of which were developed at JHU/APL, is used in the reconstruction procedure. The heart of the procedure is the Simultaneous Perturbation Stochastic Approximation (SPSA). Under restricted conditions conventional gradient techniques have had limited success at reconstructing tomography maps [7]. Unlike conventional gradient techniques, SPSA can reconstruct conductivity maps even when the gradient data are inaccurate or contaminated with noise. Under most field conditions, one should expect and be prepared to deal with measurement inaccuracies, as well as noise in the data. The success of the SPSA 
algorithm is attributed to its ability to reconstruct even under extreme conditions of noise and inaccuracies in the input parameters and boundary conditions.

Another practical advantage of the ECOL technique is that the current can be injected into the soil from a location that is away from the area of interest or where mines are presumed present. However, the technique is limited by the need to insert the electrodes into the soil for measuring the potential.

\section{Acknowledgments}

This work was supported by the JHU/APL Independent Research and Development Program, Environmental Thrust Area. Mr. P. R. Zarriello helped in field measurements. The editor for this chapter is Mr. D. Portwood.

\section{References}

1. Spall, J. C., 1988, "A Stochastic Approximation Algorithm for Large-Dimensional System in the Kiefer-Wolfowitz Sitting," Proceedings of IEEE Conference in Decision Control, pp. 1544-1548.

2. Spall, J. C., 1992, "Multivariate Stochastic Approximation Using a Simultaneous Perturbation Gradient Approximation," IEEE Transactions On Automatic Control, Vol. 37, pp. 332-341.

3. Brown, B. H., D. C. Barber, W. Wang, Liquin Lu, A. D. Leathard, R. H. Smallwood, A. R. Hampshire, R. Mackay, and K. Hatzigalanis, 1994, "Multi-Frequency Imaging and Modeling of Respiratory Related Electrical Impedance Changes," Physiol. Meas., Vol. 15, A1-A12.

4. Brown, B. H., D. C. Barber, A. H. Morice, and A. D. Leathard, 1994, "MultiFrequency Imaging and Modeling of Respiratory Related Electrical Impedance Changes," IEEE Trans. Biomed. Eng., Vol. 41, No. 8, pp. 729-733.

5. Smith, R. W., I. L. Freeston, and B. H. Brown, 1995, "A Real-Time Electrical Impedance Tomography System for Clinical Use- Design and Preliminary Results," IEEE Trans. Biomed. Eng., Vol. 42, No. 2, pp. 133-140, and references therein.

6. Wexler, A., B. Fry, and M. R. Neuman, 1985, "Impedance-Computed Tomography Algorithm and System," Applied Optics, Vol. 24, No. 23, pp. 3985-3993.

7. Cook, R. D., G. J. Saulnier, D. G. Gisser, J. C. Goble, J. C. Newell, and D. Isaacson, 1994, "ACT3: A High-Speed, High-Precision Electrical Impedance Tomograph," IEEE Trans. Biomed. Eng., Vol. 41, No. 8, pp. 713-722, and references therein.

8. K. Bathe (Ed.), Finite Element Procedures in Engineering Analysis, The Southeast Book Company, Prentice-Hall, Inc., New Jersey, 1982.

9. Chin, D. C., 1994, "A More Efficient Global Optimization Algorithm Based on Styblinski and Tong," Neural Networks, Vol. 7, No. 2, pp. 573-574. 